\title{
Alignment of Nonspherical Active Particles in Chaotic Flows
}

\author{
M. Borgnino, ${ }^{1}$ K. Gustavsson, ${ }^{2}$ F. De Lillo, ${ }^{1}$ G. Boffetta, ${ }^{1}$ M. Cencini@,${ }^{3, *}$ and B. Mehlig ${ }^{2}$ \\ ${ }^{1}$ Dipartimento di Fisica and INFN, Università di Torino, via P. Giuria 1, 10125 Torino, Italy \\ ${ }^{2}$ Department of Physics, Gothenburg University, 41296 Gothenburg, Sweden \\ ${ }^{3}$ Istituto dei Sistemi Complessi, CNR, via dei Taurini 19, 00185 Rome, Italy and INFN, sezione Roma2 "Tor Vergata"
}

(Received 24 May 2019; revised manuscript received 2 August 2019; published 27 September 2019)

\begin{abstract}
We study the orientation statistics of spheroidal, axisymmetric microswimmers, with shapes ranging from disks to rods, swimming in chaotic, moderately turbulent flows. Numerical simulations show that rodlike active particles preferentially align with the flow velocity. To explain the underlying mechanism, we solve a statistical model via the perturbation theory. We show that such an alignment is caused by correlations of fluid velocity and its gradients along particle paths combined with fore-aft symmetry breaking due to both swimming and particle nonsphericity. Remarkably, the discovered alignment is found to be a robust kinematical effect, independent of the underlying flow evolution. We discuss its possible relevance for aquatic ecology.
\end{abstract}

DOI: 10.1103/PhysRevLett.123.138003

Active particles, such as motile microorganisms or artificial microswimmers, swim in a surrounding flow, either externally imposed or self-generated. In addition to transporting the active particles, velocity gradients change their swimming direction by exerting a shape-dependent torque $[1,2]$. The complex interplay of flow advection, particle orientation, and self-propulsion is fundamental to understand key processes in aquatic ecology [3-7], active matter modeling [8-10], and nano- or microtechnology with application to drug delivery $[11,12]$.

Even simple laminar steady flows give rise to intriguing phenomena when combined with self-propulsion [13-16]. Rod-shaped motile bacteria are expelled by vortices [13] and display complex trajectories in pipe flows [14]. Microfluidic experiments in shear flows found that bacteria tumble in high shear regions, causing accumulation and chemotactic depletion [15]. In shear flows, a different tumbling mechanism traps bottom-heavy gyrotactic phytoplankton [17]. It has recently been found that individual bacteria in a steady porous flow can orient their swimming direction with the local velocity, leading to a strong enhancement (depletion) of the dispersion along (transverse to) the mean flow direction [18].

The behavior of active particles in unsteady flows is considerably less explored. Gyrotactic swimmers form small-scale fractal patches in turbulence [19-21], sampling different flow regions depending on their shape [21,22]. Elongated swimmers, such as bacteria, remain quite homogeneously distributed in turbulent flows, while their orientation tends to nematically align with the vorticity [20,23], similarly to elongated tracers $[24,25]$. Much less is known about their orientation with respect to the flow velocity, which is key to light scattering in aquatic environments [26,27], and for the encounter rates between organisms [4].
For instance, flow reorientation of elongated prey in the feeding currents of predators can strongly modify the capture rates [28]. Moreover, flow-induced changes in the swimming direction can strongly alter chemotaxis, as found in steady shear flows [15].

In this Letter, aiming to fill this gap, we investigate the dependence of the orientation statistics of active particles on their shape and speed in unsteady, moderately turbulent, and stochastic flows. We find that swimming directions preferentially align with or against the local velocity field, depending on the particle shape. Solving, by means of perturbative methods, the problem with a stochastic velocity field, we trace back the origin of such an alignment to the correlation between the flow velocity and its gradients along the particle path.

We consider dilute suspensions, disregarding any form of particle interaction. In this limit, we can neglect flow modifications induced by the active particles. We model a microswimmer as a small, neutrally buoyant, nonspherical, axisymmetric particle swimming with constant speed $v_{s}$, in the direction $\boldsymbol{n}$ of its symmetry axis. Assuming the particle size is smaller than the smallest flow scale, the particle center of mass $\boldsymbol{x}$ evolves as [2]

$$
\dot{\boldsymbol{x}}=\boldsymbol{u}(\boldsymbol{x}, t)+v_{s} \boldsymbol{n},
$$

$\boldsymbol{u}(\boldsymbol{x}, t)$ being the fluid velocity at the particle position. The particle orientation rotates in response to velocity gradients, according to Jeffery's dynamics [1]

$\dot{\boldsymbol{n}}=[\mathbb{O}(\boldsymbol{x}, t)+\Lambda \mathbb{S}(\boldsymbol{x}, t)] \boldsymbol{n}-\Lambda[\boldsymbol{n} \cdot \mathbb{S}(\boldsymbol{x}, t) \boldsymbol{n}] \boldsymbol{n} \equiv \boldsymbol{J}(\boldsymbol{n})$,

where $\mathbb{O}$ and $\mathbb{S}$ are the antisymmetric (vorticity) and symmetric (strain) components of the velocity gradient 

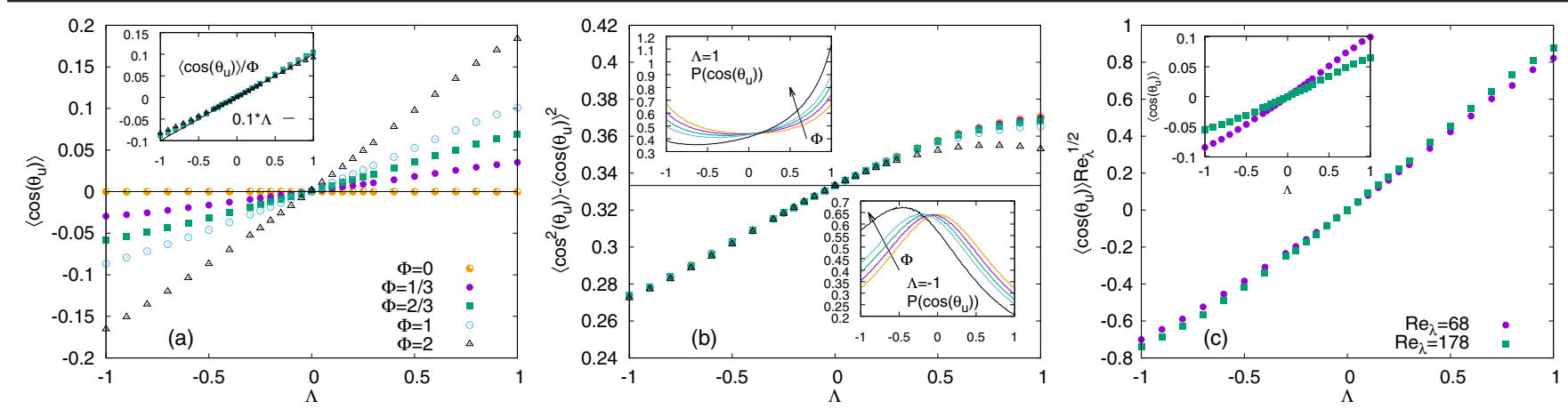

FIG. 1. Statistics of particle orientation with respect to the flow velocity, obtained from DNS of the NSE (3), as a function of the particle shape parameter $\Lambda$, for different swimming number $\Phi$. (a) $\left\langle\cos \theta_{u}\right\rangle$ vs $\Lambda$ for different $\Phi$ at $\operatorname{Re}_{\lambda} \approx 68$. Inset: $\left\langle\cos \theta_{u}\right\rangle / \Phi$ vs $\Lambda$; the solid line represents a linear best fit. (b) Variance of $\cos \theta_{u}$ vs $\Lambda$ for different $\Phi$. The top (bottom) inset shows the PDF of $\cos \theta_{u}$ for rodlike (disklike) particles with $\Phi$ from 0 to 2 along the arrows. (c) The same as (a) for $\Phi=1$ and $\operatorname{Re}_{\lambda}=68$ and 178 . The main panel shows $\left\langle\cos \theta_{u}\right\rangle \operatorname{Re}_{\lambda}^{1 / 2}$ vs $\Lambda$. The inset shows the nonrescaled data. Data are obtained by averaging over 100 snapshots, separated by about half the large-scale eddy turnover time, with up to $3 \times 10^{5}$ particles for each $\Lambda$ and $\Phi$ value.

matrix $\mathbb{A}_{i j}=\partial u_{i} / \partial x_{j}$, respectively. For $v_{s}=0$, the above dynamics reproduces that of spheroidal tracers that have recently gathered much attention [24,25,29].

The dynamics is controlled by two dimensionless numbers. The first is the shape parameter $\Lambda=\left(a^{2}-b^{2}\right) /\left(a^{2}+b^{2}\right)$ ( $a$ and $b$ being the particle size along and perpendicularly to the symmetry axis, respectively): $\Lambda=0$ for spheres, and $\Lambda= \pm 1$ for infinitely slender rods and thin platelets, respectively. The second is the swimming number $\Phi=v_{s} \tau / \ell, \ell$ and $\tau$ being the flow typical scale and time, respectively, discussed below. For $\Lambda>0$, Eqs. (1) and (2) represent a minimal model for a smooth swimming (not tumbling) bacterium $[15,16]$. Rotational diffusivity in Eq. (2) is neglected to reduce the number of parameters.

We start considering homogeneous, isotropic turbulent flows obtained by direct numerical simulations (DNS) of the Navier-Stokes equations (NSE)

$$
\partial_{t} \boldsymbol{u}+\boldsymbol{u} \cdot \nabla \boldsymbol{u}=-\nabla p+\nu \Delta \boldsymbol{u}+\boldsymbol{F},
$$

where fluid density is scaled to unity, pressure $p$ ensures flow incompressibility $(\nabla \cdot \boldsymbol{u}=0)$, and $\nu$ is the viscosity. The stirring force $\boldsymbol{F}$ is an incompressible, zero-mean, temporally uncorrelated Gaussian random field, injecting kinetic energy at large scales at a rate $\epsilon$ to generate a statistically steady state. We solve Eqs. (3) by means of a 2/3-dealiased pseudospectral solver with a second-order Runge-Kutta scheme, in a triply periodic domain with $N^{3}=128^{3}-512^{3}$ mesh points. The Kolmogorov length $\eta=\left(\nu^{3} / \epsilon\right)^{1 / 4}$ is larger than the grid spacing, and the time step much smaller than the Kolmogorov timescale, $\tau_{\eta}=(\nu / \epsilon)^{1 / 2}$, to well resolve the small-scale dynamics. The velocity and its gradients at particle positions, needed to integrate Eqs. (1) and (2), are obtained via a third-order interpolation scheme. The swimming number is defined as $\Phi=v_{s} \tau_{\eta} / \eta=v_{s} / u_{\eta}, u_{\eta}$ being the Kolmogorov velocity.
We consider moderately turbulent flows, with Taylor-scale Reynolds number $\operatorname{Re}_{\lambda}=\sqrt{15} u_{\text {rms }} /(\nu \epsilon)^{1 / 2} \approx 70-180$.

For nonspherical particles, we find a remarkable alignment of the swimming direction with the local velocity field quantified by the statistics of the angle, $\theta_{u}$, between $\boldsymbol{n}$ and $\boldsymbol{u}$. Figure 1(a) shows that $\left\langle\cos \theta_{u}\right\rangle \neq 0$ provided the particle is not spherical $(\Lambda \neq 0)$ and active $(\Phi>0)(\langle[\cdots]\rangle$ denotes the average over particle positions). The data suggest that $\left\langle\cos \theta_{u}\right\rangle \propto \Lambda \Phi$ [inset in Fig. 1(a)] at least for small $\Phi$ and $\Lambda$, with some deviations from linear behavior for $|\Lambda| \rightarrow 1$. We remark that such an alignment depends on the particle shape: It is "polar-like" for elongated particles $(\Lambda>0)$ and antipolar for disklike ones $(\Lambda<0)$. Thus, on average, rodlike particles swim along the underlying flow velocity, while disklike ones against it.

The variance of $\cos \theta_{u}$ [Fig. 1(b)] displays a nontrivial dependence on $\Lambda$, while it is almost insensitive to $\Phi$, except around $\Lambda \rightarrow 1$, where it slightly decreases with $\Phi$. The different behavior for disklike (rodlike) particles reflects qualitative differences in the probability density function (PDF) of $\cos \theta_{u}$. For disks (bottom inset), the PDF of $\cos \theta_{u}$ displays a peak that gradually moves from 0 (swimming normal to velocity) to negative values at increasing $\Phi$. Conversely, for rods (top inset), the PDF is bimodal at \pm 1 for $\Phi=0$ with a progressive bias in favor of the +1 peak at increasing $\Phi$. Thus, elongated particles $(\Lambda>0)$ align with the local fluid velocity for any $\Phi$, but the alignment changes from nematic to polar upon increasing $\Phi$.

To rationalize the above observations, we now consider a statistical model for the velocity field, $\boldsymbol{u}(\boldsymbol{x}, t)$, which allows for analytical treatments. As detailed in Ref. [30] (see Sec. I A in Supplemental Material [31]), we consider a (single scale, single time) random Gaussian velocity field parametrized by typical flow speed $u_{f}$ with correlation length $\ell_{f}$ and time $\tau_{f}$. We introduce the additional dimensionless number, namely, the Kubo number $\mathrm{Ku}=u_{f} \tau_{f} / \ell_{f}$, quantifying how rapidly the fluid velocity fluctuates. 


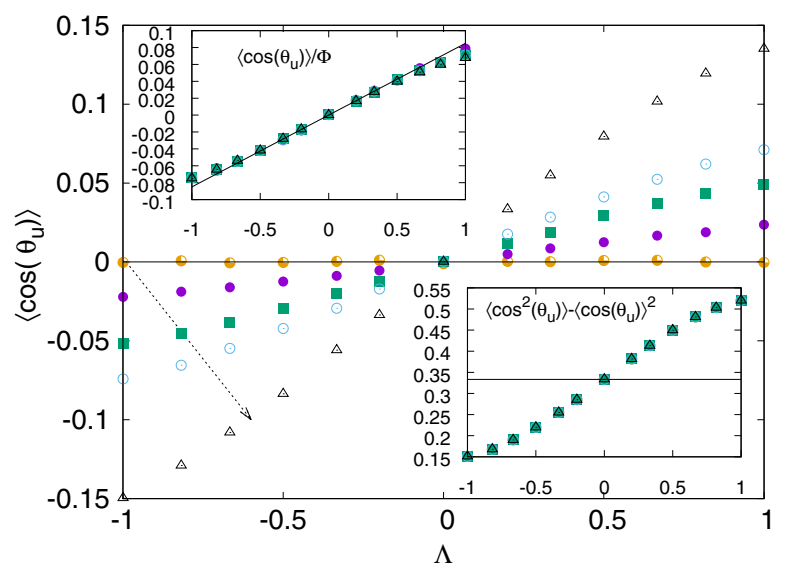

FIG. 2. Statistics of orientation obtained by simulations of the stochastic model for $\mathrm{Ku}=10$. Main panel: $\left\langle\cos \theta_{u}\right\rangle$ vs $\Lambda$ for different swimming speeds $\Phi_{s}=0,0.3,0.7,1,2$ as indicated by the arrow. Top inset: $\left\langle\cos \theta_{u}\right\rangle / \Phi_{s}$ vs $\Lambda$. Bottom inset: Variance of $\cos \theta_{u}$.

Figure 2 displays the statistics of alignment obtained from a numerical simulation of the stochastic model. The agreement with the results obtained in turbulent flows (Fig. 1) is remarkable, demonstrating that the alignment is a robust kinematical phenomenon, i.e., independent of the dynamics producing the flow. This is in contrast with the known alignment observed for elongated swimmers with the local vorticity $\boldsymbol{\omega}(\boldsymbol{x}, t)=\nabla \times \boldsymbol{u}(\boldsymbol{x}, t)[20,23]$, which is absent in the stochastic flow. Indeed, the origin of alignment with vorticity is dynamical as discussed in Ref. [25] for elongated tracers and stems from the formal similarity of Eq. (2) for $\Lambda=1$ with the Lagrangian dynamics of vorticity. See Sec. IV in Supplemental Material [31] for further considerations.

The advantage of the stochastic model is that it allows for reaching an analytical understanding of the basic mechanism for the alignment. In particular, we study the statistics of $(\boldsymbol{n} \cdot \boldsymbol{u})$ instead of $(\boldsymbol{n} \cdot \boldsymbol{u}) /|\boldsymbol{u}|=\cos \theta_{u}$, as they convey the same qualitative information on alignment (Fig. 3) and are easier to handle. The main difficulty in analyzing Eqs. (1) and (2) lies in their nonlinear dependence on the particle position. Such a hindrance can be overcome in the perturbation theory, by iteratively improving approximations for the particle trajectory, a technique successfully employed to analyze inertial particles [30] and gyrotactic swimmers [21]. This corresponds to an expansion in the Kubo number [30].

In the following, we briefly outline the main steps; detailed calculations can be found in Sec. II A of Supplemental Material [31]. To apply the perturbation theory, we introduce dimensionless variables with $t=t^{\prime} \tau_{f}, x=x^{\prime} \ell_{f}$, and $u=u^{\prime} u_{f}$, in terms of which Eqs. (1) and (2) read $\dot{\boldsymbol{x}}^{\prime}=\mathrm{Ku} \boldsymbol{u}^{\prime}+\Phi_{s} \boldsymbol{n}$ and $\dot{\boldsymbol{n}}=\mathrm{Ku} \boldsymbol{J}^{\prime}(\boldsymbol{n})$, respectively, with swimming number $\Phi_{s}=v_{s} \tau_{f} / \ell_{f}$. The above equations imply that, for $\mathrm{Ku}=0$, the particle paths are simply
$\boldsymbol{x}_{t^{\prime}}^{\prime(d)}=\boldsymbol{x}_{0}^{\prime}+\Phi_{s} \boldsymbol{n}_{0} t^{\prime}$, where $(d)$ denotes the zeroth-order (deterministic) solution. We can now write $\boldsymbol{x}_{t^{\prime}}^{\prime}=\boldsymbol{x}_{t^{\prime}}^{\prime(d)}+$ $\delta \boldsymbol{x}_{t^{\prime}}$ and expand Eqs. (1) and (2) to the desired order in $\delta \boldsymbol{x}_{t^{\prime}}$, leading to an expansion in $\mathrm{Ku}$ at fixed $\Phi_{s}[30,31]$. The result is then averaged using the known correlation functions of the (Gaussian) velocity field and its derivatives. Using flow isotropy, homogeneity, and incompressibility, the stationarystate average of the scalar product between $\boldsymbol{n}$ and $\boldsymbol{u}$ takes the (dimensional) form

$$
\langle\boldsymbol{n} \cdot \boldsymbol{u}\rangle=-\left.d \Lambda \int_{0}^{t} d t_{1} \partial_{R} C_{\|}\left(R, t_{1}\right)\right|_{\boldsymbol{R}=\boldsymbol{x}_{t_{1}}^{(d)}},
$$

$d$ being the spatial dimension and $R=|\boldsymbol{R}|$. For the stochastic flow, the longitudinal velocity covariance takes the form $C_{\|}(R, t) \equiv\langle[\boldsymbol{u}(\boldsymbol{x}+\boldsymbol{R}, t) \cdot \hat{\boldsymbol{R}}][\boldsymbol{u}(\boldsymbol{x}, 0) \cdot \hat{\boldsymbol{R}}]\rangle=$ $\exp \left[-\left(R^{2} / 2 \ell_{f}^{2}+|t| / \tau_{f}\right)\right] / d$. Substituting it in Eq. (4) and using $\boldsymbol{x}_{t_{1}}^{(d)}=\Phi_{s} \boldsymbol{n}_{0} t_{1}$, the integral can be easily computed (see Sec. II C in Supplemental Material [31]), yielding for $\Phi_{s} \ll 1$

$$
\langle\boldsymbol{n} \cdot \boldsymbol{u}\rangle \simeq u_{f} \Lambda \mathrm{Ku} \Phi_{s},
$$

which agrees well with the numerically obtained scaling of $\cos \theta_{u}$ in terms of $\Lambda$ and $\Phi$ [inset in Fig. 1(a) and Fig. 2]. Figure 3(a) shows that, for $\mathrm{Ku} \ll 1$, statistical-model simulations perfectly agree with the theoretical prediction.

Neglecting vorticity in Eq. (2), in the limit of small swimming speeds and $|\Lambda|$, an expansion similar to that used in Ref. [37] can be performed, yielding (see Sec. V in Supplemental Material [31])

$$
\langle\boldsymbol{n} \cdot \boldsymbol{u}\rangle=\frac{2 \Lambda v_{s}}{d+2} \int_{0}^{t} d t_{1} t_{1} \operatorname{Tr}\left\langle\mathbb{S}\left(\boldsymbol{x}_{t_{1}}^{L}, t_{1}\right) \mathbb{S}\left(\boldsymbol{x}_{0}^{L}, 0\right)\right\rangle,
$$

which expresses $\langle\boldsymbol{n} \cdot \boldsymbol{u}\rangle$ in terms of the correlation function of the strain along Lagrangian trajectories, $\boldsymbol{x}_{t}^{L}$, i.e., corresponding to the dynamics (1) with $v_{s}=0$. Note that the above expression, being free from any assumption on the flow statistics, requires only $v_{s}$ and $\Lambda$ to be small and should, therefore, be valid for generic flows and Kubo numbers (see Sec. V in Supplemental Material [31]). We measured $\operatorname{Tr}\left\langle\mathbb{S}\left(\boldsymbol{x}_{t}^{L}, t\right) \mathbb{S}\left(\boldsymbol{x}_{0}^{L}, 0\right)\right\rangle$ along Lagrangian trajectories in DNS and numerically computed the integral in Eq. (6), obtaining a prediction for $\langle\boldsymbol{n} \cdot \boldsymbol{u}\rangle$ that agrees well with the numerical data, at least for not too large $\Phi$ and $\Lambda$ [Fig. 3(b)].

The physical meaning of Eq. (4) is as follows: The alignment results from the nonzero correlation between the velocity and its gradients at different times [this is $\left.\partial_{R} C_{\|}\left(R, t-t_{1}\right)\right]$. However, such a correlation brings a nonzero contribution only if swimming $\left(v_{s} \neq 0\right)$ and nonsphericity $(\Lambda \neq 0)$ are present. Essentially, swimming in the instantaneous direction breaks the fore-aft symmetry, and 

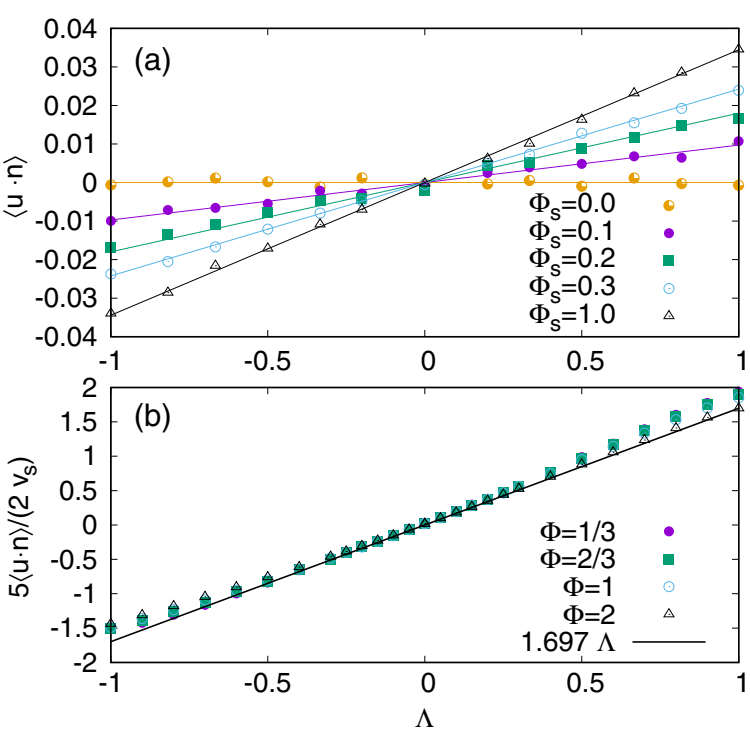

FIG. 3. Comparison between theoretical predictions and simulations for both the statistical model and DNS. (a) $\langle\boldsymbol{n} \cdot \boldsymbol{u}\rangle$ vs $\Lambda$ for different $\Phi_{s}$ obtained numerically for the statistical model with $\mathrm{Ku}=0.1$ (symbols) compared with the theoretical prediction (4) (solid lines). (b) $5\langle(\boldsymbol{n} \cdot \boldsymbol{u})\rangle /\left(2 v_{s}\right)$ vs $\Lambda$ for different $\Phi=$ $v_{s} / u_{\eta}$ for the $\mathrm{DNS}$ at $\operatorname{Re}_{\lambda} \approx 68$. The solid line represents the prediction (6); $1.697 \Lambda$ with the numerical prefactor was numerically obtained evaluating the strain correlation function along tracer trajectories.

the dynamics of $\boldsymbol{n}$ is no longer identical to the dynamics of $\boldsymbol{- n}$, leading to a nonzero value for $\langle\boldsymbol{n} \cdot \boldsymbol{u}\rangle$ (see Sec. II C in Supplemental Material [31] for further considerations). For the second moment, the situation is different; to the order of $\mathrm{Ku}^{2}$ and for $\Phi \ll 1$, we find (see Sec. III in Supplemental Material [31])

$\left\langle(\boldsymbol{n} \cdot \boldsymbol{u})^{2}\right\rangle / u_{f}^{2} \sim \frac{1}{d}+\frac{\mathrm{Ku}^{2} \Lambda}{d}+\frac{\mathrm{Ku}^{2} \Phi^{2} \Lambda}{2 d}(2 d \Lambda-11)$,

which depends on $\Lambda$ also for nonswimming particles, as confirmed by simulations.

In the statistical model, there is a single timescale for both the velocity and its gradients. Conversely, in turbulence, there is a timescale separation between them controlled by $\operatorname{Re}_{\lambda} \approx T / \tau_{\eta}$ ( $T$ being the integral timescale). Thus, for strong turbulence, the dynamics of the orientation, ruled by velocity gradients, will vary over timescales $\left(\sim \tau_{\eta}\right)$ much faster than the correlation time of the velocity $(\sim T)$, possibly depleting the alignment. This is confirmed in the inset in Fig. 1(c), showing that $\left\langle\cos \theta_{u}\right\rangle \sim \operatorname{Re}_{\lambda}^{-1 / 2}$. This $\operatorname{Re}_{\lambda}^{-1 / 2}$ scaling can be rationalized as follows. The statistical model calculations predict that, for a given $\mathrm{Ku}$, alignment depends only on $\Lambda$ and $\Phi_{s}$. Thus, we need to map the swimming parameter of the model on that used in turbulence. Following Ref. [21] (see also Sec. IA in Supplemental Material [31]), the statistical model length $\left(\ell_{f}\right)$ and time $\left(\tau_{f}\right)$ scales should be related to the Taylor length scale, $\lambda \propto u_{\mathrm{rms}} \tau_{\eta}$ and $\tau_{\eta}$, respectively, being the scales relevant to the gradients. Therefore, the swimming number to be used to compare DNS with the statistical model should be based on the rms velocity; indeed, $\Phi_{s}=$ $v_{s} \tau_{\eta} / \lambda \propto v_{s} / u_{\text {rms }}$ (see also [22] for related considerations), while we used $\Phi=v_{s} / u_{\eta}$. The two swimming numbers are thus related by $\Phi_{s} \propto \Phi\left(u_{\eta} / u_{\mathrm{rms}}\right) \propto \operatorname{Re}_{\lambda}^{-1 / 2} \Phi$, which explains the scaling observed in Fig. 1(c). DNS results (not shown) confirm that for fixed $\Phi_{s}$ the alignment statistics is independent of $\operatorname{Re}_{\lambda}$. Thus, alignment can be important also for high $\operatorname{Re}_{\lambda}$ flows, provided the particle speed is a fraction of the large-scale velocity. Such large speeds can be attained by swimmers larger than the Kolmogorov scale, for which Eqs. (1) and (2) may still be valid, provided the Stokes number defined on the particle scale is small enough, as recently found in finite-size fibers [38].

In general, alignment is expected to be important whenever turbulence is moderate, i.e., in velocity fields with not too separated scales of motion, as commonly found in environmental, laboratory, and biomedical fluids. In marine environments with calm water, the Kolmogorov velocity is on the order of $u_{\eta} \approx 300-1000 \mu \mathrm{m} / \mathrm{s}$ [4], while bacterial speeds range in $v_{s} \approx 30-300 \mu \mathrm{m} / \mathrm{s}$ [39]; consequently, $\Phi \approx 0.05-1$. Hence, depending on the Reynolds number, the alignment can be substantial. Alignment could be relevant to models for light scattering in aquatic environments [26,27], especially considering that most motile microorganisms are elongated [26], and for the encounter rates of aquatic microorganisms [4,28]. Furthermore, analogously to the findings in steady shear flows [15], flow reorientation may alter the chemotactic efficiency. For instance, flow-induced alignment could be particularly relevant to marine bacteria, many of which perform a run-reverse cycle in which the orientation is unchanged while the swimming velocity is reversed [40].

Preliminary studies, to be discussed elsewhere, show that alignment persists also in the presence of a nonhomogeneous mean flow. In this case, alignment may dramatically impact the dispersal properties along and transverse to the mean flow similarly to what is observed in steady porous flows [18], with implications for groundwater filtration and remediation and biomedical fluids. Remarkably, the experiments in Ref. [18] demonstrated preferential alignment of the bacterial swimming direction with the local flow in analogy with our findings. It would be then interesting to study alignment in the limit of steady flows, i.e., in the $\mathrm{Ku} \rightarrow \infty$ limit, to understand whether the physical mechanism for alignment is the same as that we found in unsteady flows. This is, however, beyond the scope of the present Letter.

Finally, we observe that nontrivial correlations between flow velocity and individual bacterial orientation have been reported in dense suspensions [41,42], where the 
self-generated flow is on the order of $\sim 50-100 \mu \mathrm{m} / \mathrm{s}$ with a correlation length of 30-100 $\mu \mathrm{m}$, while bacteria swim at a speed of $\sim 15-20 \mu \mathrm{m} / \mathrm{s}$ with a size of $\sim 2 \mu \mathrm{m}[41,43]$. With swimming numbers on the order of $\approx 0.15-0.4$, it is tempting to speculate that the alignment here discussed could be an important effect. However, this needs to be tested, because steric and hydrodynamic interactions, here neglected, play a major role.

In summary, we found that (disklike) rodlike active particles swimming in a moderately turbulent background flow tend to preferentially align their swimming direction (anti)parallel to the underlying flow velocity. We showed that such an alignment has a kinematical origin and analytically found its roots in the time correlations between the velocity and its gradients along particle paths together with the fore-aft symmetry breaking induced by swimming. Our study expands on the possible nontrivial behaviors of microswimmers in an external flow [5,7] from the simple cases of pipe or shear flows $[13,14]$ to realistic unsteady turbulent and chaotic flows.

We acknowledge useful discussions with R. Stocker. M. B., G. B., and F.D.L. acknowledge support by the Departments of Excellence grant (MIUR). B. M. and K. G. acknowledge support by the Knut and Alice Wallenberg Foundation, Grant No. KAW 2014.0048, and Vetenskapsrådet, Grants No. 2017-3865 and No. 201803974. G. B., M. C., F.D. L., and M.B. acknowledge CINECA under the INFN-Cineca Agreement No. INF18-fldturb and under the ISCRA initiative for the availability of high performance computing resources and support.

*Corresponding author massimo.cencini@cnr.it

[1] G. B. Jeffery, Proc. R. Soc. A 102, 161 (1922).

[2] T. J. Pedley and J. O. Kessler, Proc. R. Soc. B 231, 47 (1987).

[3] R. H. Luchsinger, B. Bergersen, and J. G. Mitchell, Biophys. J. 77, 2377 (1999).

[4] T. Kiørboe, A Mechanistic Approach to Plankton Ecology (Princeton University, Princeton, NJ, 2008).

[5] R. Rusconi and R. Stocker, Curr. Opin. Microbiol. 25, 1 (2015)

[6] J. R. Taylor and R. Stocker, Science 338, 675 (2012).

[7] J. S. Guasto, R. Rusconi, and R. Stocker, Annu. Rev. Fluid Mech. 44, 373 (2012).

[8] M. C. Marchetti, J.-F. Joanny, S. Ramaswamy, T. B. Liverpool, J. Prost, M. Rao, and R. A. Simha, Rev. Mod. Phys. 85, 1143 (2013).

[9] C. Bechinger, R. Di Leonardo, H. Löwen, C. Reichhardt, G. Volpe, and G. Volpe, Rev. Mod. Phys. 88, 045006 (2016).

[10] J. Elgeti, R. G. Winkler, and G. Gompper, Rep. Prog. Phys. 78, 056601 (2015).

[11] R. Dreyfus, J. Baudry, M. L. Roper, M. Fermigier, H. A. Stone, and J. Bibette, Nature (London) 437, 862 (2005).
[12] B. J. Nelson, I. K. Kaliakatsos, and J. J. Abbott, Annu. Rev. Biomed. Eng. 12, 55 (2010).

[13] C. Torney and Z. Neufeld, Phys. Rev. Lett. 99, 078101 (2007).

[14] A. Zöttl and H. Stark, Phys. Rev. Lett. 108, 218104 (2012); Eur. Phys. J. E 36, 4 (2013).

[15] R. Rusconi, J. S. Guasto, and R. Stocker, Nat. Phys. 10, 212 (2014).

[16] G. Junot, N. Figueroa-Morales, T. Darnige, A. Lindner, R. Soto, H. Auradou, and E. Clément, Europhys. Lett. 126, 44003 (2019).

[17] W. M. Durham, J. O. Kessler, and R. Stocker, Science 323, 1067 (2009).

[18] A. Dehkharghani, N. Waisbord, J. Dunkel, and J. S. Guasto, Proc. Natl. Acad. Sci. U.S.A. 116, 11119 (2019).

[19] W. M. Durham, E. Climent, M. Barry, F. De Lillo, G. Boffetta, M. Cencini, and R. Stocker, Nat. Commun. 4, 2148 (2013).

[20] C. Zhan, G. Sardina, E. Lushi, and L. Brandt, J. Fluid Mech. 739, 22 (2014).

[21] K. Gustavsson, F. Berglund, P. R. Jonsson, and B. Mehlig, Phys. Rev. Lett. 116, 108104 (2016).

[22] M. Borgnino, G. Boffetta, F. De Lillo, and M. Cencini, J. Fluid Mech. 856, R1 (2018).

[23] N. Pujara, M. Koehl, and E. Variano, J. Fluid Mech. 838, 356 (2018).

[24] G. A. Voth and A. Soldati, Annu. Rev. Fluid Mech. 49, 249 (2017).

[25] A. Pumir and M. Wilkinson, New J. Phys. 13, 093030 (2011).

[26] W. E. Clavano, E. Boss, and L. Karp-Boss, in Oceanography and Marine Biology: An Annual Review, edited by R. N. Gibson, R. J. A. Atkinson, and J. D. M. Gordon (Taylor \& Francis, 2007), Vol. 45, pp. 1-38.

[27] Marcos, J. R. Seymour, M. Luhar, W. M. Durham, J. G. Mitchell, A. Macke, and R. Stocker, Proc. Natl. Acad. Sci. U.S.A. 108, 3860 (2011).

[28] A. W. Visser and P. R. Jonsson, J. Plankton Res. 22, 761 (2000).

[29] M. Byron, J. Einarsson, K. Gustavsson, G. Voth, B. Mehlig, and E. Variano, Phys. Fluids 27, 035101 (2015).

[30] K. Gustavsson and B. Mehlig, Adv. Phys. 65, 1 (2016).

[31] See Supplemental Material at http://link.aps.org/ supplemental/10.1103/PhysRevLett.123.138003 for a full description of the statistical model, for details on the perturbative calculations, and for results on the alignment with vorticity, which includes Refs. [32-36].

[32] G. Falkovich, K. Gawędzki, and M. Vergassola, Rev. Mod. Phys. 73, 913 (2001).

[33] K. Gustavsson, M. Z. Sheikh, D. Lopez, A. Naso, A. Pumir, and B. Mehlig, New J. Phys. 21, 083008 (2019).

[34] U. Frisch, Turbulence (Cambridge University Press, Cambridge, England, 1995), p. 296.

[35] E. Calzavarini, R. Volk, M. Bourgoin, E. Lévêque, J.F. Pinton, and F. Toschi, J. Fluid Mech. 630, 179 (2009).

[36] K. Gustavsson and B. Mehlig, Europhys. Lett. 96, 60012 (2011). 
[37] S. Vajedi, K. Gustavson, B. Mehlig, and L. Biferale, J. Fluid Mech. 798, 187 (2016).

[38] D. Bakhuis, V. Mathai, R. A. Verschoof, R. Ezeta, D. Lohse, S. G. Huisman, and C. Sun, Phys. Rev. Fluids 4, 072301 (2019).

[39] G. M. Barbara and J. G. Mitchell, FEMS Microbiol Ecol. 43, 99 (2003).

[40] R. Stocker and J. R. Seymour, Microbiol. Mol. Biol. Rev. 76, 792 (2012).
[41] A. Sokolov, I. S. Aranson, J. O. Kessler, and R. E. Goldstein, Phys. Rev. Lett. 98, 158102 (2007).

[42] S. D. Ryan, G. Ariel, and A. Beer, Biophys. J. 111, 247 (2016).

[43] C. Dombrowski, L. Cisneros, S. Chatkaew, R. E. Goldstein, and J. O. Kessler, Phys. Rev. Lett. 93, 098103 (2004). 